\title{
Experiências vivenciadas no programa de extensão "Atividade física funcional e recreativa para terceira idade" por discentes do curso de fisioterapia
}

\author{
Experiences lived in the extension program "Functional and recreational physical activity for \\ the elderly" by students from the physiotherapy course
}

\author{
Milene Carrara Carmo Garcia ${ }^{1}$ \\ Michelle Marques do Vale ${ }^{2}$ \\ Jhonatan Carlos Terencio Ribeiro ${ }^{3}$ \\ Angelo Piva Biagini ${ }^{4}$
}

\section{RESUMO}

Este relato objetiva descrever as experiências vividas por duas estudantes de fisioterapia participantes da oficina de "Treinamento Funcional Terapêutico" do programa de extensão "Atividade Física Funcional e Recreativa para Terceira Idade" (AFRID), oferecido na Faculdade de Educação Física e Fisioterapia da Universidade Federal de Uberlândia, no primeiro semestre de 2019. Relata o planejamento das atividades físicas propostas aos idosos, como a rotina dos encontros realizados, assim como os objetivos esperados de cada etapa e exercício proposto e a reação e feedback tanto dos participantes quanto das estudantes. Ressalta também a importância da realização de projetos de atividade física orientada para a terceira idade objetivando a manutenção da capacidade física funcional e colaborando assim para a melhoria da qualidade de vida dos idosos. Além disso, o projeto contribuiu para a vivência, construção e aplicação de conhecimentos práticos acerca da fisioterapia aplicada à geriatria, as necessidades observadas nessa população no âmbito do exercício físico, assim como os benefícios e desafios encontrados.

Palavras-chave: Envelhecimento. Qualidade de vida. Desempenho físico funcional. Idosos.

\section{ABSTRACT}

This report aims to describe the experiences of two students of the physiotherapy course participating in the "Functional Therapeutic Training" of the extension project, "Functional and Recreational Physical Activity for the Elderly" (AFRID) at the Federal University of Uberlândia, in the first semester of 2019. It reports how the planning of physical activities proposed to the elderly was carried out, how the routine of the meetings was carried out, as well as the expected objectives of each stage and proposed exercises and the reaction and feedback of both participants and students. It also emphasizes the importance of carrying out physical activity projects aimed at the elderly, aiming at maintaining physical capacity and thus collaborating to improve the quality of life of the elderly. In addition, it describes how the project contributed to the experience, construction and application of practical knowledge about physiotherapy applied to geriatrics, the necessities observed in this population in the scope of physical exercise, as well as the benefits and challenges encountered by students.

\footnotetext{
1 Graduanda em Fisioterapia na Universidade Federal de Uberlândia, Minas Gerais, Brasil (milenecarrara02@gmail.com).

2 Graduanda em Fisioterapia na Universidade Federal de Uberlândia, Minas Gerais, Brasil (michellevale@hotmail.com).

3 Mestrando em Fisioterapia na Universidade Federal do Triângulo Mineiro, Minas Gerais, Brasil (jhonatanterencio15@gmail.com).

4 Doutor em Ciências Médicas pela Universidade de São Paulo, São Paulo, Brasil; professor adjunto na Universidade Federal de Uberlândia, Minas Gerais, Brasil (pivaab@gmail.com).
} 
Keywords: Aging. Quality of life. Physical functional performance. Elderly.

\section{INTRODUÇÃO (onde estávamos...)}

Segundo o Instituto Brasileiro de Geografia e Estatística (IBGE, 2007), o aumento de idosos na população brasileira é decorrente da transição demográfica no Brasil, que tem sido caracterizada pelo envelhecimento populacional. Consequência dessa transição é o aumento de doenças crônicas não transmissíveis, ocasionando comprometimento da qualidade de vida. Dentre os fatores que comprometem a qualidade de vida, destaca-se a capacidade funcional, definida por Valério e Ramos (2013, p. 3) como "habilidade de executar tarefas físicas, preservação das atividades mentais e uma situação adequada de integração social”.

Nesse sentido, conforme afirma Montenegro e Silva (2007 apud MATSUNO; MATSUNO 1991), é necessário que a condição funcional do idoso seja preservada e/ou recuperada com o objetivo de devolver qualidade de vida e autonomia à realização de atividades cotidianas. Avaliações realizadas por profissionais capacitados permitem definir estratégias que visam à promoção de saúde de idosos.

De acordo com Rikli e Jones (1999), a discussão acerca da capacidade funcional originou-se da aptidão física, uma vez que esse conceito, em idosos, está diretamente relacionado à execução autônoma das atividades diárias. Assim, é interessante destacar os componentes da aptidão física, que incluem força, flexibilidade, coordenação motora, equilíbrio e agilidade. Considerando que esses componentes naturalmente entram em declínio durante o processo de envelhecimento, eles são apontados como essenciais, pois, ao serem mantidos, podem contribuir para a manutenção da independência, minimizar os riscos de queda e retardar a perda de velocidade na execução de atividades cotidianas. (GOBBI, 1997)

A força muscular é um dos domínios de maior importância para que haja uma boa qualidade de vida. No entanto, sabe-se que a fraqueza dos músculos pode avançar a ponto de impedir que o idoso realize atividades básicas da vida diária. Além disso, em condições normais, o desempenho da força apresenta o seu auge entre 20 e 30 anos, após esse período permanece relativamente estável ou diminui ligeiramente. Ainda, segundo Tartaruga et al. (2005 apud FLECK; KRAEMER, 1999), a força muscular diminui aproximadamente 15\% durante os 60 e 70 anos e depois, aproximadamente, $30 \%$, e depende de fatores:

Existem diversos fatores que contribuem para a perda da força muscular com a idade. Alterações musculoesqueléticas, acúmulo de doenças crônicas, 
medicamentos necessários para o tratamento de doenças preexistentes, alterações no sistema nervoso, redução das secreções hormonais, desnutrição e atrofia por desuso são os principais fatores. (TARTARUGA et al., 2005, p. $1)$.

O equilíbrio é outro domínio que possui relação diretamente proporcional com a qualidade de vida do idoso, pois, em decorrência do processo de envelhecimento, o controle postural eficiente e a integridade osteoarticular são comprometidos. Além disso, a presença de déficit auditivo em decorrência do envelhecimento do sistema vestibular pode causar alterações no equilíbrio efetivo do idoso, ocasionando tonturas (MACIEL; GUERRA, 2005).

A flexibilidade, de acordo com Sartori, Sartori e Bagnara (2012, p. 1) é a "capacidade física responsável pela execução voluntária de um movimento de amplitude angular máxima” e, apesar de diminuir com o envelhecimento devido ao encurtamento da musculatura, é considerada um fator determinante para a qualidade de vida e realização de atividades cotidianas. Seu declínio gera dificuldade para agachar, pouca mobilidade articular e dores generalizadas. De acordo com os autores, a realização de atividade física envolvendo amplitude de movimento, acima dos habituais, tem repercussões benéficas no sistema esquelético e muscular, assim como aumenta a independência e autonomia.

Do mesmo modo, conforme afirma Ferreira e Gobbi (2003), as alterações inerentes ao envelhecimento interferem na capacidade de combinar movimentos, influenciando na coordenação e agilidade, componentes que se influenciam e são influenciados pelos outros componentes na construção de uma ação global mais eficiente.

A agilidade parece ser uma das perdas funcionais mais prevalentes e pode ser definida como a capacidade de realizar movimentos de curta duração e alta intensidade com mudanças de direção ou alterações na altura do centro de gravidade do corpo. (MADEIRAS et al., 2015 apud ZAGO; GOBBI, 2003).

O treinamento funcional consiste em estimulação do sistema neuromuscular objetivando aumento da capacidade funcional. No caso dos idosos, a estruturação de programas com esse objetivo é baseada na funcionalidade, muitas vezes com o uso de exercícios e movimentos considerados funcionais para as necessidades específicas da vida diária do idoso. Portanto, é um método fundamental para a prevenção e/ou recuperação de incapacidades que são atribuídas ao processo de envelhecimento, além de melhora do sistema psicobiológico (NETO et al., 2016). 
A formação dos futuros profissionais de fisioterapia no campo da gerontologia deve ser completa, tanto com a fundamentação teórica apresentada na estrutura do curso (disciplina obrigatória) quanto na participação em atividades práticas, como por exemplo, em projetos de extensão (MENEZES; SOUZA; CARDOSO, 2007). Assim, o AFRID contribui de forma relevante para a formação dos discentes envolvidos que passam a compreender a importância da promoção de saúde para a população idosa quando envolvida em programas que oferecem atividade física funcional.

Segundo o Plano Nacional de Extensão Universitária (2012), a extensão é um processo educativo, cultural e científico. Por meio de atividades de pesquisa e projetos sociais, além de exercer a responsabilidade social, a extensão proporciona o contato do aluno com o mundo e através dessa realidade ele poderá complementar seu aprendizado, se tornando, portanto, um mecanismo de aprendizagem e uma atividade produtora de conhecimento (GOULART, 2004; NUNES; SILVA, 2011).

O presente relato objetiva descrever as experiências vividas por duas estudantes de fisioterapia participantes da oficina de "Treinamento Funcional Terapêutico" do programa de extensão AFRID, oferecido na Faculdade de Educação Física e Fisioterapia da Universidade Federal de Uberlândia, no primeiro semestre de 2019.

\section{METODOLOGIA (o que nos marcou...)}

A oficina "Treinamento Funcional Terapêutico", atividade oferecida pelo AFRID, assiste idosos de Uberlândia-MG, a fim de proporcionar-lhes melhor qualidade de vida. Todas as ações desenvolvidas dentro do programa, bem como a publicação de seus respectivos resultados seguem o preconizado pela Resolução nº 466/2012.

Antes do ingresso nas oficinas do AFRID, foram realizadas avaliações funcionais dos idosos inscritos, com o objetivo de analisar seus perfis físicos e funcionais. Os testes realizados para avaliar os idosos foram: Teste de Sentar e Levantar em 30 segundos para avaliar a força de membros inferiores; Teste Timed Up and Go, que avalia a mobilidade e equilíbrio dinâmico do idoso; Teste de flexibilidade de Wells, que avalia a flexibilidade da cadeia posterior do tronco; Teste de Força de Preensão Palmar, para predispor força muscular global, além dos questionários sobre medo de cair, qualidade de vida e depressão. 
A obtenção de dados como força, coordenação motora, equilíbrio, agilidade e flexibilidade são essenciais para melhor conhecer aqueles futuros participantes. Caso os idosos apresentassem baixo desempenho nas avaliações funcionais, independente do domínio, durante a oficina de treinamento funcional era trabalhado o domínio com maior ênfase, a fim de melhorar sua condição e, consequentemente a qualidade de vida do idoso.

Ao longo do $1^{\mathrm{o}}$ semestre de 2019, foram realizados encontros semanais entre os idosos e os extensionistas. Os encontros ocorriam três vezes por semana (segundas, quartas e sextas) no período da manhã. Para oferecer assistência de melhor qualidade, bem como estabelecer vínculo, o grupo foi dividido em dois, com horários distintos. Turma $\mathrm{A}$ das $7 \mathrm{~h} 30 \mathrm{~min}$ às $8 \mathrm{~h} 30 \mathrm{~min}$ e turma $\mathrm{B}$ das $8 \mathrm{~h} 30 \mathrm{~min}$ às $9 \mathrm{~h} 30 \mathrm{~min}$.

A rotina estabelecida na oficina propunha inicialmente a aferição da pressão arterial do idoso. Esta ação permitia às extensionistas verificar se o indivíduo se apresentava em condições adequadas (hemodinamicamente) a participar das atividades propostas. Caso o indivíduo estivesse com a pressão acima dos parâmetros normais, ele era orientado a permanecer sentado confortavelmente e, posteriormente sua pressão arterial seria aferida (o Colégio Americano de Medicina do Esporte recomenda maiores cuidados e acompanhamento médico na realização de atividade física de indivíduos que apresentam valores de pressão arterial acima de $140 \mathrm{mmHg} / 90 \mathrm{mmHg}$, uma vez que a prática física tem a capacidade de aumentar ainda mais a pressão arterial, possibilitando acidentes cardiovasculares). Em seguida, os idosos eram orientados a realizar uma caminhada com velocidade moderada a rápida por 10 minutos e, posteriormente, era incluído nesse percurso (caminhada) o subir e descer escadas, assim, essa etapa constituía o aquecimento (AMERICAN COLLEGE..., 2014).

A etapa seguinte oferecia atividades que contemplavam domínios como força muscular, equilíbrio, coordenação motora, agilidade e flexibilidade. Orientados pelo monitor da oficina, cabia às extensionistas selecionar os materiais que seriam utilizados e acompanhar a execução das atividades pelos idosos. Destacamos ainda que todas as atividades propostas eram discutidas entre as extensionistas e o monitor responsável. As atividades propostas mais comumente são descritas no quadro 1.

Os exercícios elaborados normalmente utilizavam equipamentos disponibilizados pelo projeto, como a bola suíça, halteres, caneleiras, colchonetes, bolas (de tamanhos variados, dependendo da atividade proposta), bastão, cones, elásticos, anilhas e cadeiras. Em exercícios que utilizavam equipamentos de peso variável, eram disponibilizados pesos diferentes para 
melhor adaptação e execução pelo idoso, cabendo às extensionistas orientá-lo quanto a melhor escolha, sempre objetivando qualidade na realização da atividade.

A oficina desenvolvia-se na forma de circuito, em que o número de exercícios propostos era igual ao número de participantes presentes no dia, definindo assim, o número de estações do circuito de treinamento, de modo com que cada um pudesse executar um exercício simultâneo aos outros participantes. O tempo em cada exercício era cronometrado e, ao final dele, todos os idosos mudavam de estação de forma que no término da oficina todos tivessem realizado todas as atividades propostas.

Após todos participantes terem passado por todas as estações do circuito, era proposta uma sessão de alongamento objetivando a recuperação da frequência cardíaca e pressão arterial (volta à calma).

Quadro 1 - Relação entre componente da aptidão física e exercício

\begin{tabular}{|c|c|c|}
\hline & Exercício & Descrição do exercício \\
\hline \multirow{4}{*}{ Equilíbrio } & Caminhar em linha reta & $\begin{array}{l}\text { Segurando um bastão à frente do corpo com os } \\
\text { membros superiores estendidos, deve-se caminhar } \\
\text { em linha reta, com um pé à frente do outro (posição } \\
\text { tandem). }\end{array}$ \\
\hline & $\begin{array}{l}\text { Equilíbrio com a bola } \\
\text { suíça }\end{array}$ & $\begin{array}{l}\text { Sentar-se na bola e levantar o membro superior e } \\
\text { inferior opostos. }\end{array}$ \\
\hline & Equilíbrio deitado & $\begin{array}{l}\text { O participante se deita no rolo (ao longo da coluna } \\
\text { vertebral) e, sem apoio, levanta os membros } \\
\text { inferiores alternados, com ou sem o uso da } \\
\text { caneleira. }\end{array}$ \\
\hline & $\begin{array}{l}\text { Super-homem de } \\
\text { joelhos }\end{array}$ & $\begin{array}{l}\text { Na posição de quatro apoios, deve-se levantar os } \\
\text { membros superior e inferior opostos e paralelos ao } \\
\text { chão. }\end{array}$ \\
\hline \multirow{2}{*}{$\begin{array}{l}\text { Coordenação e } \\
\text { agilidade }\end{array}$} & & $\begin{array}{l}\text { O participante deve levantar um dos membros } \\
\text { inferiores e aproximar o pé de cones pequenos } \\
\text { dispostos no chão, afastados, em uma sequência } \\
\text { específica. }\end{array}$ \\
\hline & & $\begin{array}{l}\text { Com o uso da escada de agilidade (no chão), o } \\
\text { participante deve realizar uma sequência de } \\
\text { movimentos o mais rápido que conseguir. }\end{array}$ \\
\hline \multirow{4}{*}{ Força (tronco) } & Prancha isométrica & $\begin{array}{l}\text { Manter o corpo paralelo ao solo durante um minuto, } \\
\text { utilizando apenas os pés e os cotovelos como base } \\
\text { de apoio. }\end{array}$ \\
\hline & Ponte & $\begin{array}{l}\text { Deitado de barriga para cima com os joelhos } \\
\text { flexionados, os pés no chão e as mãos ao longo do } \\
\text { corpo, o idoso realizava elevação e abaixamento de } \\
\text { quadril. }\end{array}$ \\
\hline & Prancha lateral & $\begin{array}{l}\text { Manter o corpo reto com o apoio apenas no } \\
\text { antebraço e a parte lateral dos pés. }\end{array}$ \\
\hline & Abdominais & $\begin{array}{l}\text { Feito com o uso de bola suíça ou não. Na posição } \\
\text { deitada e com os pés no chão (joelhos flexionados), }\end{array}$ \\
\hline
\end{tabular}




\begin{tabular}{|c|c|l|}
\hline \multirow{2}{*}{$\begin{array}{c}\text { Força (membro } \\
\text { superior) }\end{array}$} & $\begin{array}{l}\text { Exercício com uso de } \\
\text { halteres (ombro) }\end{array}$ & $\begin{array}{l}\text { deve-se elevar o tronco (como se para posicionar-se } \\
\text { na posição sentada), trabalhando os músculos } \\
\text { abdominais. }\end{array}$ \\
\cline { 2 - 3 } & $\begin{array}{c}\text { Exercício com uso de } \\
\text { halteres (cotovelo) }\end{array}$ & Abdução/Adução e Flexão/Extensão. \\
\hline \multirow{3}{*}{$\begin{array}{c}\text { Força (membro } \\
\text { inferior) }\end{array}$} & Sentar e levantar & $\begin{array}{l}\text { Com os membros superiores cruzados no peito, } \\
\text { deve-se sentare levantar da cadeira sem apoio. }\end{array}$ \\
\cline { 2 - 4 } & Agachamento & $\begin{array}{l}\text { Feito com o uso da bola suíça ou anilha. A partir da } \\
\text { posição inicial (de pé, com os pés afastados) o } \\
\text { participante deve flexionar o quadril e o joelho, } \\
\text { jogando o quadril para trás, como se estivesse } \\
\text { "sentando" }\end{array}$ \\
\cline { 2 - 3 } & $\begin{array}{c}\text { Exercícios com o uso da } \\
\text { caneleira }\end{array}$ & \begin{tabular}{l} 
Abdução de quadril (em posição deitada ou em pé). \\
\hline
\end{tabular} \\
\hline
\end{tabular}

\section{RESULTADOS E DISCUSSÃO (e então...)}

Os idosos participantes da oficina de treinamento funcional apresentaram média de idade de 67,4 anos, sendo 18 mulheres e 3 homens. Na distribuição por sexo, seguindo o que a literatura tem mostrado, as mulheres são mais adeptas a participar de atividades fora de sua casa. Destacamos também que uma grande parcela dos participantes apresentava boas condições físicas. Eram ativos, relatando que há algum tempo participavam de programas/atividades físicas oferecidas pela FAEFI, com destaque para o Programa AFRID.

Nossa participação como extensionistas do AFRID, permitiu-nos perceber que os idosos estão cada vez mais em busca da manutenção da sua qualidade de vida, autonomia e independência. Desse modo, justifica-se a necessidade deles estarem inseridos em programas que ofereçam atividade física. Acreditamos que essa mentalidade é decorrente da grande disseminação de informações na atualidade sobre saúde e bem-estar. Assim, passamos a entender nosso papel social, ou seja, disseminar informações.

Num primeiro momento, pensávamos que iríamos trabalhar com uma população frágil e resistente às atividades pensávamos em propor, ideia que se atrelava à possível idade dos participantes. Porém, já nas primeiras conversas, essa imagem foi quebrada. Pelo contrário, em geral, os participantes se mostravam dispostos na realização das atividades, com bom humor e dedicação, fatores que contribuíam bastante para o bom relacionamento que estabelecemos com os participantes, além da calorosa interação entre eles. Os próprios idosos propunham modificações nos exercícios que, caso fossem pertinentes, eram adotadas pelo 
monitor e por nós extensionistas, a fim de contribuir para melhor adequação e desempenho dos praticantes.

Verificamos, no início das atividades, que os idosos tinham mais dificuldades em realizar as atividades propostas, uma vez que relataram estar sem executar atividades físicas por um espaço de tempo considerável. No entanto, com o decorrer e a evolução das atividades propostas, eles relataram melhora na qualidade de vida, e já conseguiam efetuar diversas tarefas cotidianas com maior destreza, como descer e subir do ônibus, sentar-se e levantar com maior facilidade, executar tarefas domésticas, dentre outras. Além disso, nos foi relatado que sentiam menos dores no decorrer do dia, que estavam perdendo peso e sentindo-se mais dispostos.

As proposições de atividades sempre foram flexíveis. Em vários momentos, houve a necessidade de readequação das atividades devido a alguns participantes apresentarem limitações ou receios em relação à realização daquelas mais desafiadoras, por exemplo, as que exigiam mais equilíbrio. Nesses casos, a ajuda física das extensionistas (como dar a mão para apoiar-se) era sempre oferecida, a fim de estimular e gerar mais confiança para realização das atividades. Comumente, em um caso particular, era requisitada a mudança de todos os exercícios que eram realizados na posição deitada (no colchonete), uma vez que o participante demonstrava receio e mal-estar ao realizá-los.

Para nós extensionistas, foi notória a dificuldade dos idosos em realizar os exercícios relacionados ao equilíbrio, revelando o medo de cair e a queixa de "tontura", presentes nessa população. Isso é justificável, de acordo com a Associação Médica Brasileira e Conselho Federal de Medicina, a queda em idosos é um problema de saúde pública, pois é o mais frequente acidente doméstico e a principal causa de morte acidental acima de 65 anos. (BUKSMAN et al., 2008). Esse dado evidencia ainda mais a importância do incentivo ao desenvolvimento do equilíbrio no grupo participante, o que era feito com propostas diversificadas e desafiadoras, além de exigir maior atenção e acompanhamento quando realizados.

Ao final do período, os idosos realizavam os exercícios com maior destreza e facilidade, em conformidade com Ruwer et al. (2005), que afirma que a queixa de tontura torna-se menos incidente nos idosos que apresentam uma vida ativa, que realizam atividade física, social e intelectual, corroborando a tese de que a prática de atividades físicas melhora o equilíbrio e, consequentemente, a qualidade de vida dos idosos (RUWER; ANGELA; SIMON, 2005). 
Durante o desenvolvimento das atividades, dia a dia, recebíamos relatos dos participantes, que durante os finais de semana faziam os exercícios em casa e, buscavam executar aqueles que ofereceram mais dificuldade durante as oficinas. Assim, eles diziam que os exercícios nos quais tinham dificuldades seriam - ou eram - praticados em casa para melhorar o desempenho nas próximas aulas. Víamos isso como engajamento e compromisso com eles próprios, pois quando discutíamos com o monitor, passamos a entender que essa atitude refletia confiança nas atividades propostas por nós, felicidade em perceber que era capaz e vínculo estabelecido.

Nesse contexto, a oferta de projetos de extensão a essa parcela da população mostra-se de grande relevância, visto que permite reduzir a incidência de doenças e agravos à saúde, decorrentes da idade avançada. Ademais, atividades físicas e recreativas são muito importantes tanto para a saúde física quanto para a saúde mental do idoso.

Imagem 1 - Realização de exercício proposto (força de membro superior)

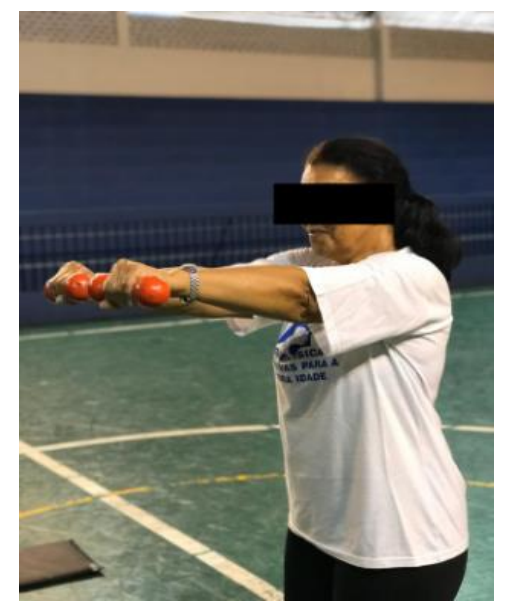

Imagem 2 - Realização de exercício proposto (força de membro superior)

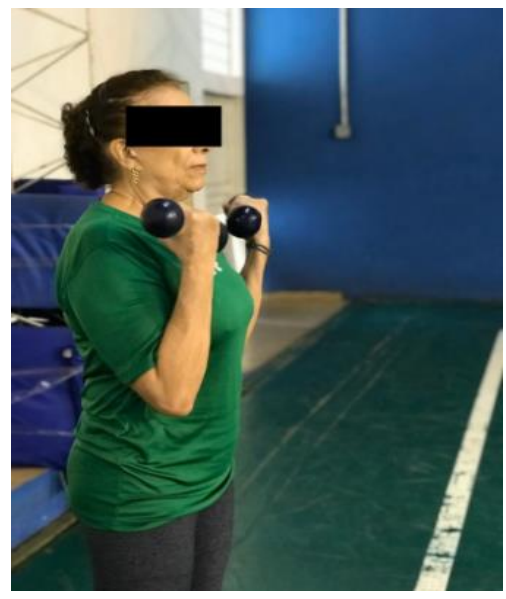

Fonte: Os autores (2019).
Imagem 3 - Realização de exercício de coordenação

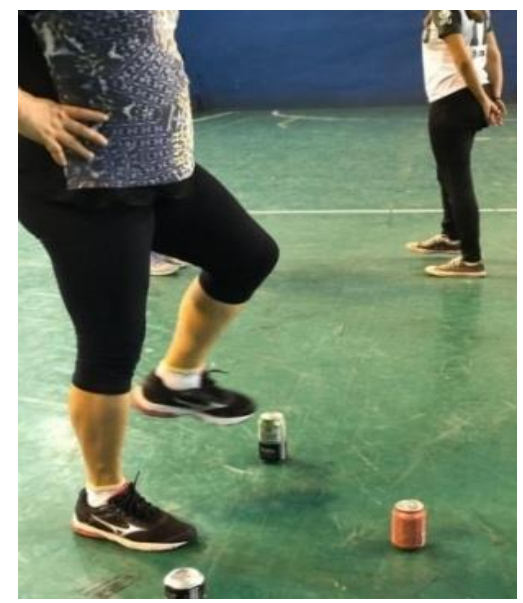


Imagem 4 - Realização do exercício de equilíbrio (andar em linha reta)

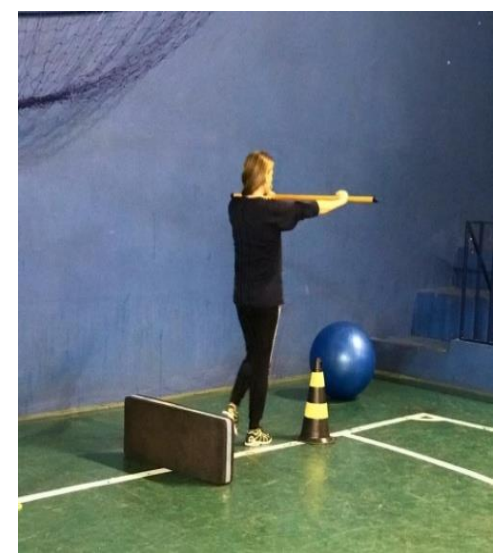

Imagem 5 - Alongamento comum aos finais de encontro

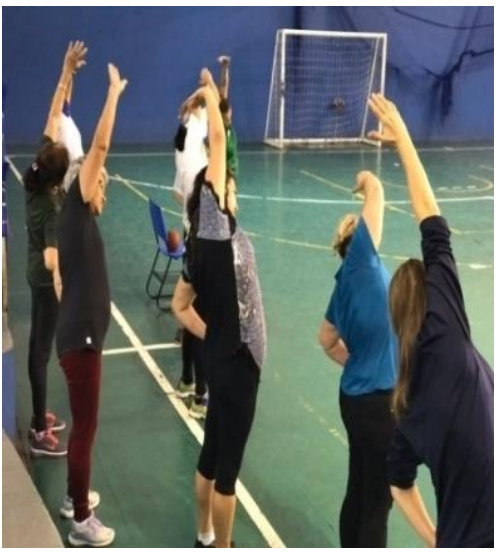

Fonte: Os autores (2019).
Imagem 6 - Alongamento realizado com equipamentos

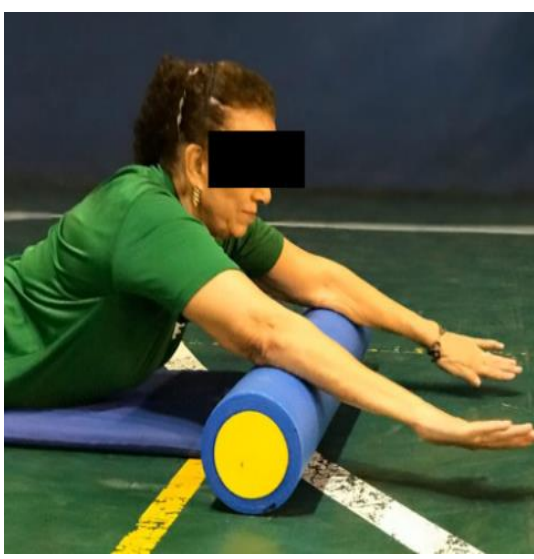

\section{CONSIDERAÇÕES FINAIS (o que ficou...)}

Ao longo do semestre, observamos e recebemos relatos positivos dos participantes, que destacavam melhora da qualidade de vida, da autoestima, da confiança em saber que conseguiriam realizar tarefas, antes realizadas com dificuldades. Esses relatos, por sua vez, nos motivavam a buscar mais (estudar) para oferecer mais.

Além dos benefícios percebidos relacionados aos participantes, é de grande importância ressaltar a contribuição do projeto a nós extensionistas por meio da vivência e prática da atuação do fisioterapeuta junto à população idosa e nas atividades em grupo. Desse modo, as alunas extensionistas integrantes da oficina de treinamento funcional fisioterapêutico, sempre recebiam explicações e, tinham a oportunidade de discutir sobre os exercícios propostos, relacionando-os com as necessidades e dificuldades da população assistida, assim como com as disciplinas ainda não cursadas no curso de fisioterapia. Portanto, evidencia-se a contribuição na construção do conhecimento prático e teórico necessários à formação de um profissional de fisioterapia.

Por fim, fica para nós extensionistas do AFRID que estimular a participação de indivíduos que envelhecem ou já são caracterizados como idosos em atividades físicas funcionais tornase essencial, pois aprendemos e vivenciamos que a população envelhece a passos largos. Condição que não significa em hipótese alguma inatividade, dependência, isolamento, depressão, mas sim uma nova fase da vida. 


\section{REFERÊNCIAS (nosso suporte...)}

AMERICAN COLLEGE OF SPORTS MEDICINE. Diretrizes do ACMS para os testes de esforço e sua prescrição. Tradução de Dilza Balteiro Pereira de Campos. 9. ed. Rio de Janeiro: Guanabara Koogan Ltda., 2014.

FERREIRA, L.; GOBBI, S. Agilidade geral e agilidade de membros superiores em mulheres de terceira idade treinadas e não treinadas. Revista Brasileira Cineantropometria Desempenho Humano, Florianópolis, v. 5, n. 1, p. 46-53, 2003.

FÓRUM DE PRÓ-REITORES DE EXTENSÃO DAS INSTITUIÇÕES PÚBLICAS DE EDUCAÇÃO SUPERIOR BRASILEIRAS. 2012, Manaus. Política Nacional de Extensão Universitária. Florianópolis: UFSC, 2015.

GOBBI, S. Atividade física para pessoas idosas e recomendações da Organização Mundial de Saúde de 1996. Revista Brasileira de Atividade Física e Saúde, Florianópolis, v. 2, n. 2, p. 41-49, 1997. Doi: 10.12820/rbafs.v.2n2p41-49.

GOULART, A. T. A importância da pesquisa e da extensão na formação do estudante universitário e no desenvolvimento de sua visão crítica. Revista Horizonte, Belo Horizonte, v. 2, n. 4, p. 60-73, jan./jun. 2004. Doi: 10.5752/P.2175-5841.2004v2n4p60.

IBGE. Síntese de Indicadores Sociais: uma análise das condições de vida da população brasileira. Disponível em: https://biblioteca.ibge.gov.br/visualizacao/livros/liv36151.pdf. Acesso em: 19 jun. 2020.

MACIEL, A. C. C.; GUERRA, R. O. Prevalência e fatores associados ao déficit de equilíbrio em idosos. Revista Brasileira de Ciência e Movimento, Brasília, v. 13, n. 1, 2005. Doi: 10.18511/rbcm.v13i1.610.

MADEIRAS, J. G. et al. Atividade física na agilidade de idosos. Revista UNINGÁ, Maringá, v. 44,4 n. $1, \quad$ jun. 2015.2 Disponível em: http://revista.uninga.br/index.php/uninga/article/view/1217. Acesso em: 19 jun. 2020.

MENEZES, R. L. de.; SOUZA, M. R.; CARDOSO, T. R. C. O conhecimento de acadêmicos de fisioterapia em relação à velhice e ao envelhecimento. Fragmentos de Cultura, Goiânia, v. 17, n. 3/4, p. 293-301, mar./abr. 2007. Doi: 10.18224/frag.v17i2.277.

MONTENEGRO, S. M. R. S.; SILVA, C. A. B. da S. Os efeitos de um programa de fisioterapia como promotor de saúde na capacidade funcional de mulheres idosas institucionalizadas. Revista Brasileira de Geriatria e Gerontologia, Rio de Janeiro, v. 10, n. 2, p. 161-178, 2007. Doi: 10.1590/1809-9823.2007.10023.

NUNES, A. L. de P. F.; SILVA, M. B. da C. A extensão universitária no ensino superior e sociedade. Mal-estar e Sociedade, Barbacena, ano IV, n. 7, p. 119-133, jul./dez. 2011. Disponível em: http://revista.uemg.br/index.php/malestar/article/view/60/89. Acesso em: 15 mar. 2020. 
RESENDE-NETO A. G. et al. Treinamento funcional para idosos: uma breve revisão. Revista Brasileira de Ciência e Movimento, Brasília, v. 24, n. 3, p. 167-177, 2016. Doi: $10.18511 / \mathrm{rbcm} . v 24 \mathrm{i} 3.6564$.

RIKLI, R. E.; JONES, C. J. Development and validation of a functional fitness test for cummunity-residing older adults. Journal of Aging and Physical Activity, v. 7, n. 2, p. 129161, 1999. Doi: 10.1123/japa.7.2.129.

RUWER, S. L.; ROSSI, A. G.; SIMON, L. F. Equilíbrio no idoso. Revista Brasileira de Otorrinolaringologia, São Paulo, v. 71, n. 3, p. 298-303, maio/jun. 2005. Doi: 10.1590/S0034-72992005000300006.

SARTORI, M. N.; SARTORI, M. R. BAGNARA, I. C. A flexibilidade e o idoso. Revista Digital de Educação Física e Desportes, Buenos Aires, ano 17, n. 169, jun. 2012. Disponível em: https://www.efdeportes.com/efd169/a-flexibilidade-e-o-idoso.htm. Acesso em: 15 mar. 2020 .

SOCIEDADE BRASILEIRA DE GERIATRIA E GERONTOLOGIA. Queda em idosos: prevenção. 2008. Disponível em: https://sbgg.org.br/wp-content/uploads/2014/10/quedaidosos.pdf. Acesso em: 20 abr. 2020.

TARTARUGA, M. P. et al. Treinamento de força para idosos: uma perspectiva de trabalho multidisciplinar. Revista Digital de Educação Física e Desportes, Buenos Aires, ano 10, n. 82, mar. 2005. Disponível em: https://www.efdeportes.com/efd82/treinam.htm. Acesso em: 10 mar. 2020.

VALERIO, M. P.; RAMOS, L. R. Promoção de atividades físicas à população idosa: revisando possibilidades. Revista Didática Sistêmica, Rio Grande, v. 15, n. 2, p. 155-173, 2013. Disponível em: https://periodicos.furg.br/redsis/article/view/4158. Acesso em: 18 abr. 2020 .

Submetido em 18 de maio de 2020.

Aprovado em 18 de junho de 2020. 